\title{
Ipertensione arteriosa, continuum cardiovascolare e ruolo clinico dei sartani
}

\author{
Maurizio Destro ${ }^{(1)}$
}

\begin{abstract}
The cardiovascular and cardiorenal continuum comprises the transition from cardiovascular risk factors to endothelial dysfunction and atherosclerosis, to chronic congestive heart failure, and-stage renal disease or premature death. RAAS (renin-angiotensin-aldosterone system) is involved in all steps along this pathway. Data from clinical studies involving valsartan and other ARBs provide evidence of the reduction of risk of cardiovascular events, and end-organ damage in the heart, kidneys and brain. This paper summarizes the status on research of ARBs based on clinical trials and regulatory approval.
\end{abstract}

Keywords: cardiovascular continuum, angiotensin II receptor blockers, hypertension Farmeconomia e percorsi terapeutici 2011; 12(Suppl 1): 3-7

\section{CONTINUUM CARDIOVASCOLARE E FISIOPATOLOGIE ASSOCIATE}

Il concetto di continuum cardiovascolare identifica una situazione nella quale una serie progressiva di eventi, partendo da uno o più fattori di rischio, evolve, se non interrotta, fino alla cardiopatia terminale e alla morte. Il sistema renina-angiotensina-aldosterone (RAAS) è implicato nello sviluppo e nella progressione del danno d'organo a livello cardiaco, vascolare, renale e cerebrale causando disfunzioni e alterazioni che portano all'aumento di morbilità e mortalità che si riscontra nei pazienti ipertesi (Figura 1).

All'inizio del processo la presenza di uno o più fattori di rischio determina, a livello vascolare, la comparsa di un'alterazione funzionale nota come disfunzione endoteliale. A causa di questa le arterie di resistenza, e in particolare i vasi coronarici, perdono progressivamente la loro capacità di rispondere a stimoli vasodilatanti sviluppando, al contrario, una tendenza a un'eccessiva risposta vasocostrittiva.

Contemporaneamente si ha il progressivo sviluppo di placche ateroscleotiche stenosanti in vario grado il lume arterioso, le cui possibili complicanze (rottura, emorragia) predispongono alla trombosi, riducendo o occludendo completamente, il lume vasale, dando origine a quell'insieme di manifestazioni cliniche denominate sindromi ischemiche acute. Queste a livello coronarico determinano la sindrome coronarica acuta (SCA), la cui evoluzione più sfavorevole è rappresentata dall'infarto miocardico acuto (IMA). A seguito di quest'ultimo possono insorgere una serie di modificazioni strutturali a carico del ventricolo sinistro indicate con il termine generale di rimodella-
(I) Direttore U.O. Medicina Interna, Ospedale di Treviglio, A.O. "Treviglio-Caravaggio"

Corresponding author Maurizio Destro Maurizio_Destro@ ospedale.treviglio.bg.it
Figura 1

Ruolo del sistema renina-angiotensinaaldosterone (RAAS) nel continuum cardiovascolare

IGT = ridotta tolleranza al glucosio; IVS = ipertrofia ventricolare sinistra 


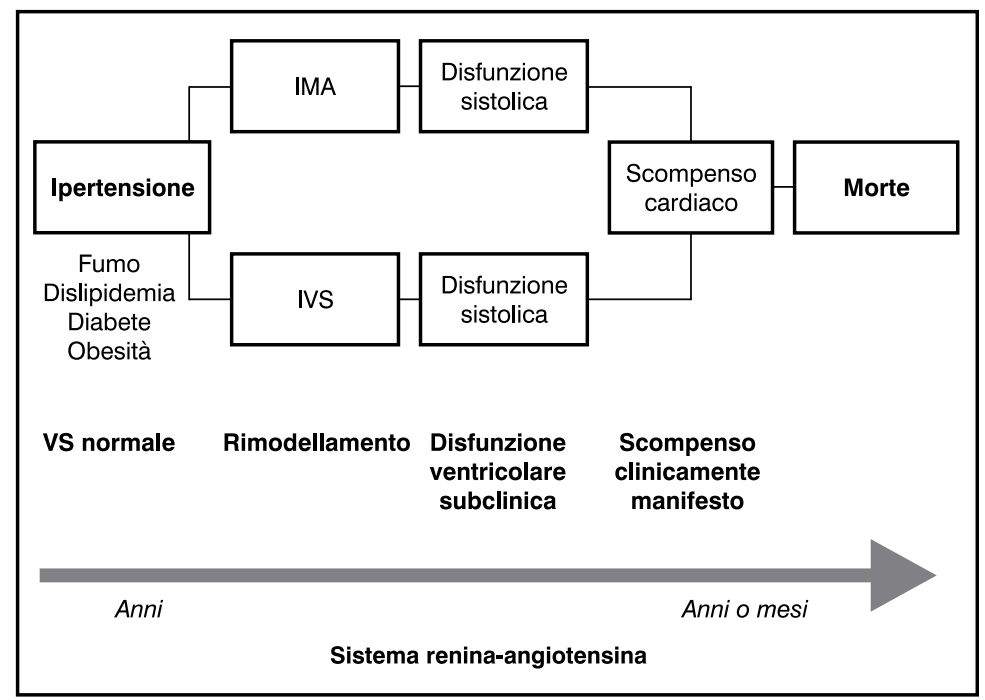

Figura 2

Ipertensione arteriosa e continuum cardiovascolare

IMA = infarto miocardico acuto; IVS = ipertrofia ventricolare sinistra $\mathrm{VS}=$ ventricolo sinistro

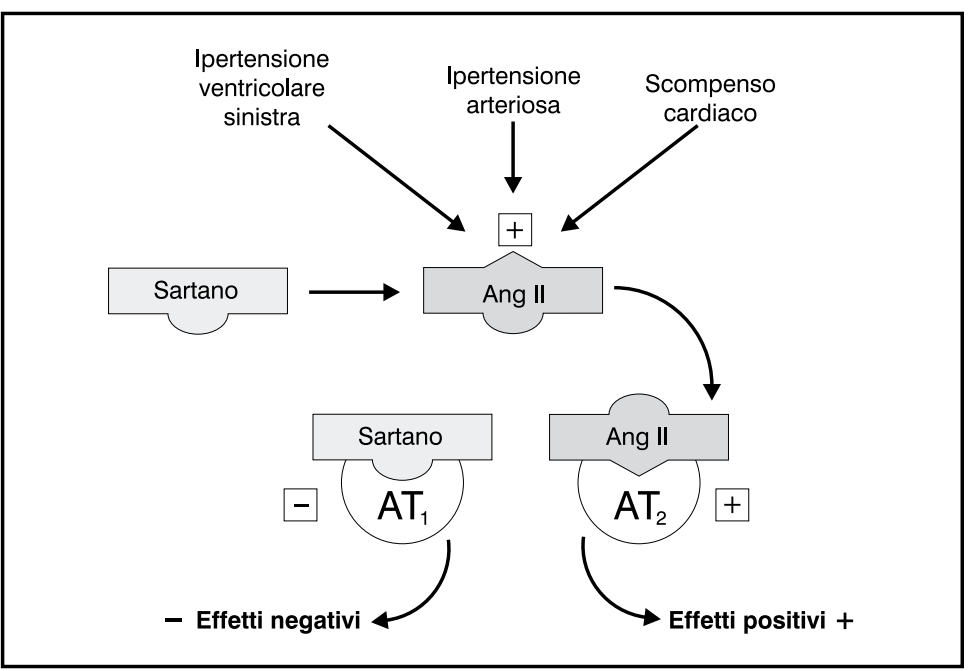

Figura 3

Azione degli antagonisti recettoriali dell'angiotensina II: blocco dei recettori AT, e stimolo indiretto dei recettori $A T_{2}$

Ang $\|$ = angiotensina $\|$ mento ventricolare con conseguente modifica della funzione sistolica, sino alla comparsa delle manifestazioni cliniche dello scompenso cardiaco. Il paziente iperteso può però giungere alla condizione di scompenso cardiaco terminale non soltanto a seguito di IMA, ma anche attraverso lo sviluppo della cardiopatia ipertensiva caratterizzata da ipertrofia ventricolare sinistra e successiva disfunzione sistolica (Figura 2).

Anche il rene rappresenta un organo bersaglio del processo aterosclerotico, con modificazioni che riguardano sia le arterie renali principali sia le arteriole intrarenali, determinando il quadro di angiosclerosi renale che, congiuntamente alla nefropatia diabetica, rappresenta la causa più frequente di sviluppo di insufficienza renale cronica e della sua progressione verso lo stadio terminale di malattia renale.

\section{MODULAZIONE DEL RAAS CON I SARTANI}

I bloccanti recettoriali dell'angiotensina II (Angiotensin Receptor Blockers, ARB), o sartani, caratterizzano una classe di farmaci cardiovascolari che interviene in maniera clinicamente rilevante lungo tutte le fasi del continuum cardiovascolare.

La modulazione del RAAS ottenibile con i sartani ha caratteristiche specifiche derivanti dal meccanismo d'azione, relativamente al quale il blocco recettoriale dell'angiotensina II può essere definito specifico, in quanto non interferisce con altri sistemi di regolazione, completo, perché si esplica indipendentemente dalle singole vie enzimatiche di generazione dell'angiotensina II e selettivo in quanto vengono demodulati prevalentemente gli effetti derivanti dall'interazione tra angiotensina II e recettore $\mathrm{AT}_{1}$. In base a quest'ultima caratteristica i sartani andrebbero definiti, per quanto riguarda l'interazione con i recettori dell'an-

\begin{tabular}{|c|c|c|c|c|c|c|c|}
\hline & $\begin{array}{l}\text { Popolazione } \\
\text { in studio }\end{array}$ & Valsartan & Losartan & Irbesartan & Candesartan & Telmisartan & Olmesartan \\
\hline & Scompenso & Val-HeFT & $\begin{array}{l}\text { ELITE-II } \\
\text { HEAAL }\end{array}$ & I-PRESERVE & CHARM & - & - \\
\hline & Ipertensione & $\begin{array}{l}\text { VALUE } \\
\text { Val-SYST } \\
\text { JIKEI }\end{array}$ & LIFE & CHOICE & $\begin{array}{l}\text { SCOPE } \\
\text { TROPHY } \\
\text { AMAZE }\end{array}$ & - & - \\
\hline & Post-IMA & $\begin{array}{l}\text { VALIANT } \\
\text { VALVACE }\end{array}$ & OPTIMAAL & - & - & - & - \\
\hline & $\begin{array}{l}\text { Diabete, } \\
\text { insufficienza } \\
\text { renale }\end{array}$ & $\begin{array}{l}\text { MARVAL I e II } \\
\text { DROP } \\
\text { SMART }\end{array}$ & RENAAL & $\begin{array}{l}\text { IDNT } \\
\text { IRMA || }\end{array}$ & $\begin{array}{c}\text { CALM } \\
\text { DIRECT }\end{array}$ & DETAIL & ROADMAP \\
\hline \multirow{2}{*}{$\begin{array}{l}\text { Tabella I } \\
\text { Grandi studi sui sartani: } \\
\text { numerosità della } \\
\text { casistica trattata }\end{array}$} & $\begin{array}{l}\text { Elevato } \\
\text { rischio cv }\end{array}$ & $\begin{array}{l}\text { NAVIGATOR } \\
\text { VAL-MARC } \\
\text { GISSI-AF }\end{array}$ & - & - & ACCESS & $\begin{array}{c}\text { TRANSCEND } \\
\text { ONTARGET } \\
\text { PRoFESS }\end{array}$ & - \\
\hline & $\begin{array}{l}\text { Totale } \\
\text { pazienti (n) }\end{array}$ & 100.000 & 22.400 & 14.600 & 17.500 & 52.000 & 4.400 \\
\hline
\end{tabular}


giotensina II, come bloccanti selettivi dei recettori $\mathrm{AT}_{1}$ e stimolanti indiretti dei recettori $\mathrm{AT}_{2}[1]$ (Figura 3).

\section{Eterogeneità della classe dei sartani}

I sartani a tutt'oggi sono oggetto di un ampio progetto di ricerca cardiovascolare volto a valutare, oltre all'efficacia antipertensiva, anche quella di inibire/ridurre la comparsa e la progressione del danno d'organo a livello cardiaco, cerebrale, renale, vascolare e metabolico. Queste molecole vanno considerate eterogenee per vari aspetti, tra cui la selettività recettoriale; valsartan, a tutt'oggi, è l'ARB dotato del più alto grado di selettività per i recettori $\mathrm{AT}_{1}[2]$ e di conseguenza è quello che meglio esercita l'aspetto più peculiare della modulazione del RAAS ottenibile con il blocco down-stream a livello recettoriale.

I risultati espressi dai grandi trial con sartani per quanto riguarda l'efficacia terapeutica evidenziano generalmente per le molecole esaminate un buon livello della stessa, ma contestualmente hanno evidenziato due sostanziali differenze tra i vari farmaci. La prima differenza è di tipo quantitativo, in quanto alcuni principi attivi sono stati estensivamente valutati mentre altri assai meno (Tabella I); la seconda è invece prettamente qualitativa in quanto la capacità $\mathrm{di}$ interrompere i vari step del continuum cardiovascolare è stata dimostrata con alcuni principi attivi e non con altri.

\section{Profilo d'efficacia e rimborsabilità dei singoli sartani}

I sartani si differenziano dunque ampiamente per quanto riguarda lo sviluppo clinico dei singoli principi attivi, in quanto le conoscenze attualmente disponibili sull'efficacia nelle specifiche condizioni patologiche che caratterizzano il continuum cardiovascolare, le posologie, le combinazioni e le titolazioni ottimali, la durata dei trattamenti e la sicurezza di impiego in termini di rapporto rischio-beneficio sono molto diverse tra loro.

L'eterogeneità della documentazione clinico-sperimentale disponibile per i singoli sartani ha importanti ricadute, sia di tipo scientifico che di tipo regolatorio. Dal punto di vista scientifico, la solidità delle conoscenze sull'efficacia terapeutica della classe ha indotto le più autorevoli società scientifiche internazionali ad aggiornare le linee guida per il trattamento delle condizioni patologiche comprese nel continuum cardiovascolare [3]. Analizzando le stesse si deduce che dichiarare un solo sartano sufficiente sia per il trattamento antipertensivo che per le comorbilità dell'ipertensione arteriosa, realizzandosi al di fuori delle indicazioni ufficiali desunte dai trial, non avrebbe sostegno clinico-scientifico. Da ciò ne deriva che anche dal punto di vista delle Autorità Regolatorie

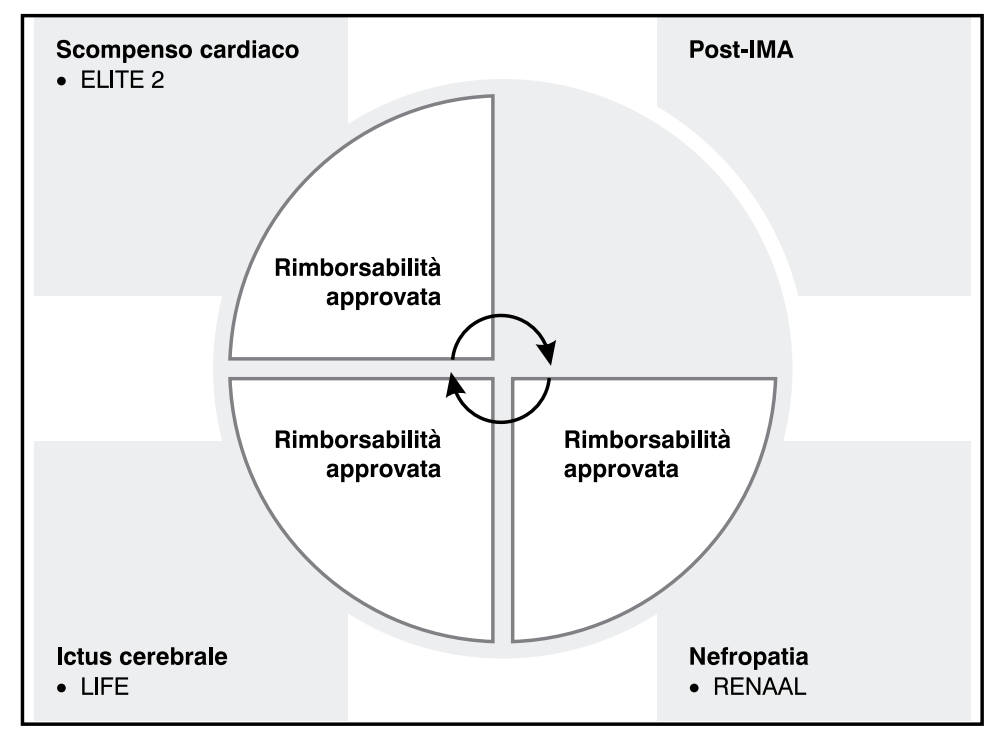

Figura 4

Circolo virtuoso di losartan: indicazioni dai trial e rimborsabilità

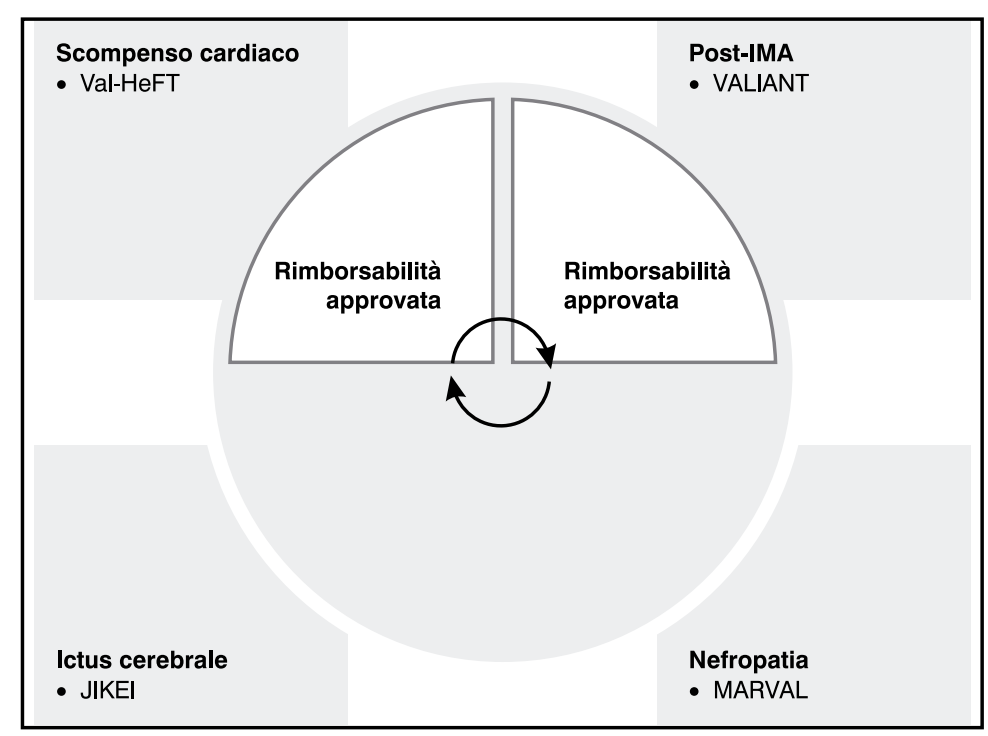

Figura 5

Circolo virtuoso di valsartan: indicazioni dai trial e rimborsabilità

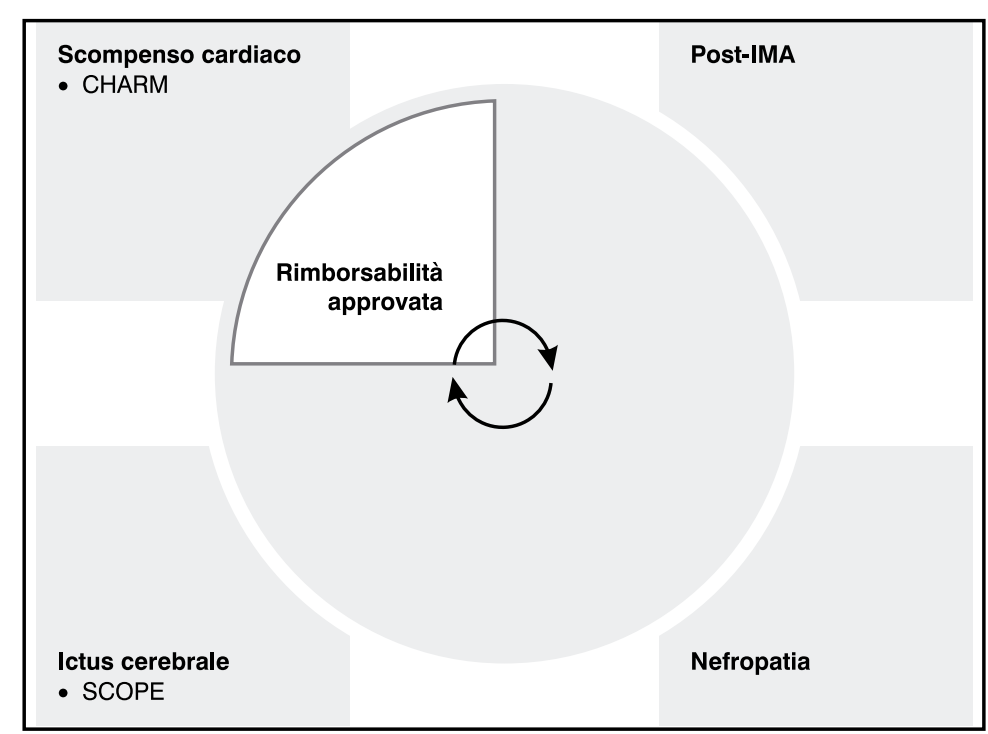

Figura 6

Circolo virtuoso di candesartan: indicazioni dai trial e rimborsabilità 


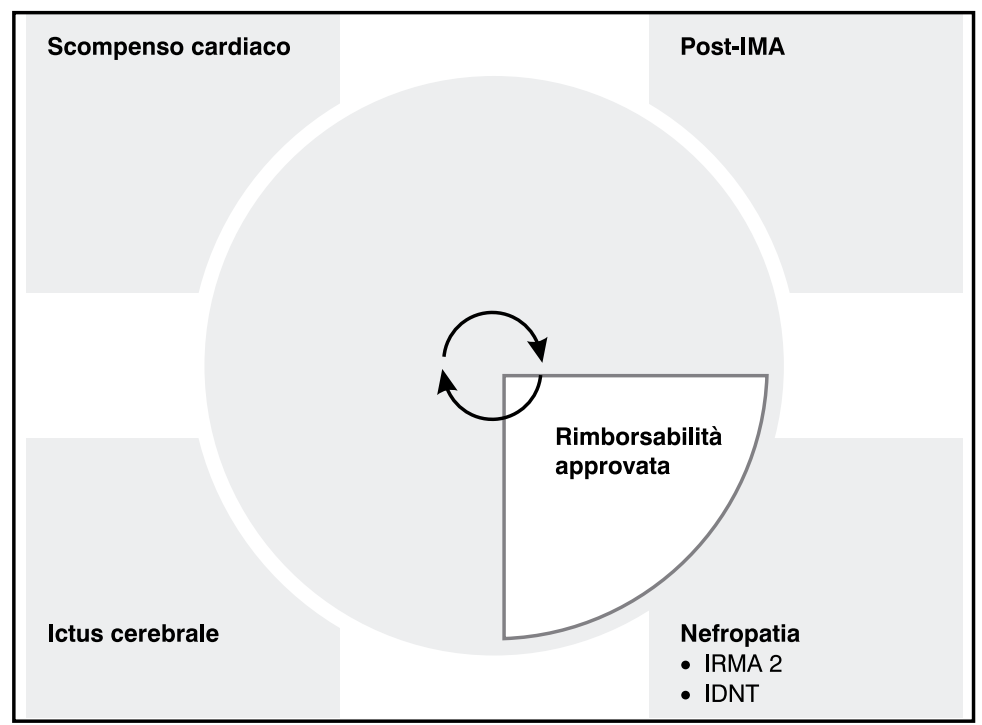

Figura 7

Circolo virtuoso di irbesartan: indicazioni dai trial e rimborsabilità

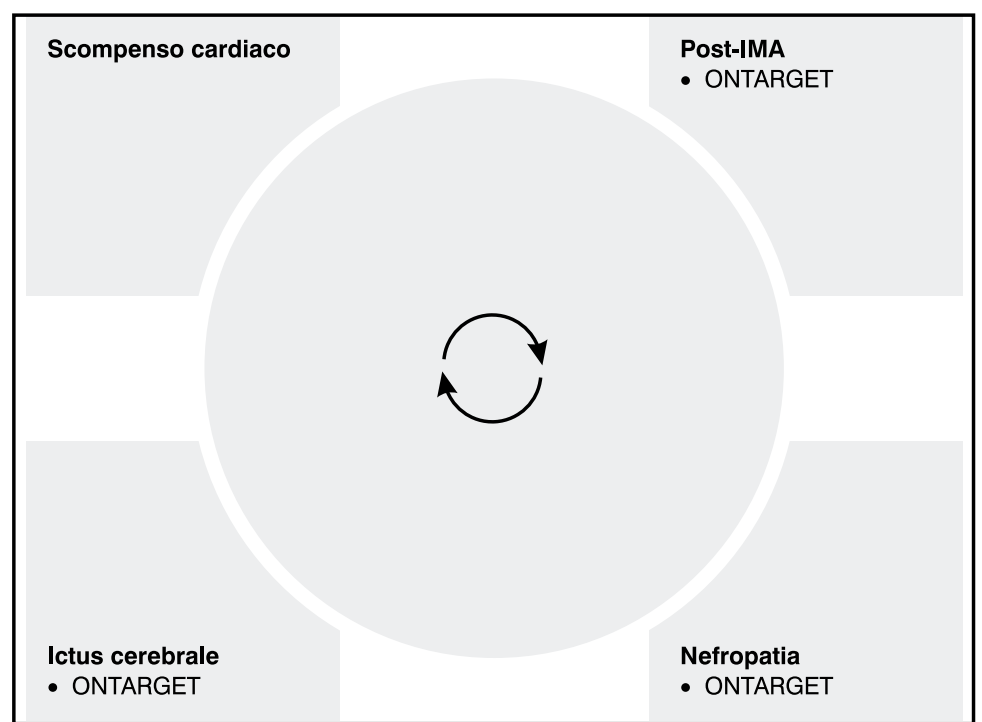

Figura 8

Circolo virtuoso di telmisartan: indicazioni dai trial e rimborsabilità

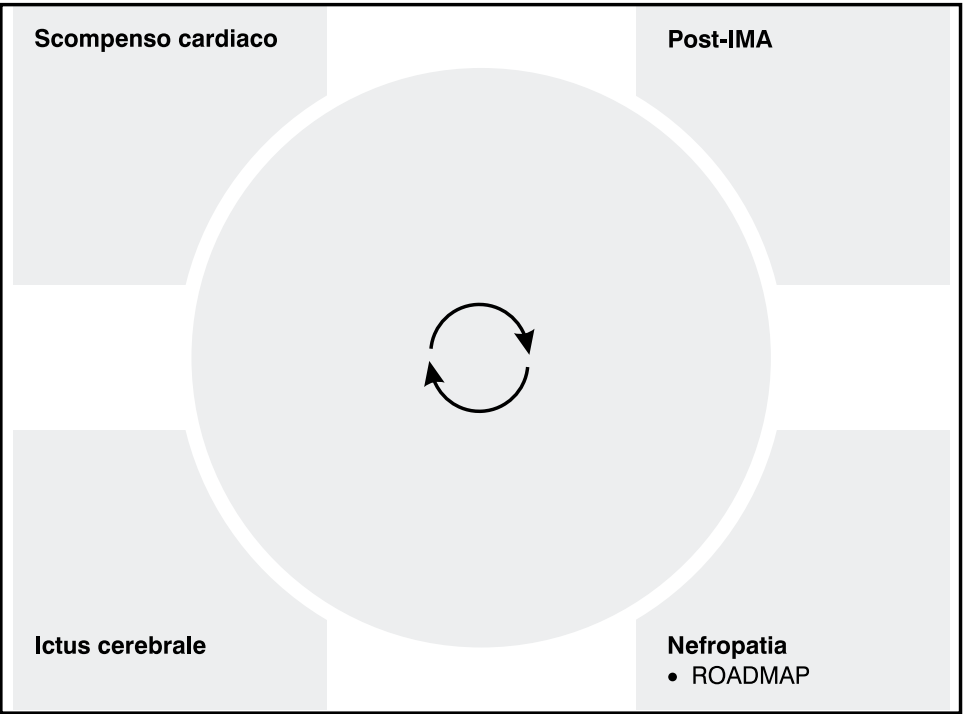

Figura 9

Circolo virtuoso di olmesartan: indicazioni dai trial e rimborsabilità tali differenze nella documentazione clinica hanno determinato un riconoscimento formale differenziato delle diverse indicazioni terapeutiche attribuite in maniera specifica alle singole molecole, sancito dalla rimborsabilità solo per alcune di esse e in specifiche condizioni di comorbilità. Conseguentemente per i medici è importante conoscere per ciascun sartano lo spettro d'impiego ottimale dimostrato e riconosciuto, che quindi può essere dedotto a partire dalle risultanze cliniche validate dalla linee guida delle Società Scientifiche Internazionali e dalle indicazioni approvate dalle Autorità Regolatorie. Ciò permette di definire per ogni sartano, alla luce delle conoscenze attualmente disponibili, oltre all'azione antiipertensiva, un profilo di efficacia ai fini della protezione degli eventi cardio-cerebro-nefrovascolari.

Le Figure 4-9 evidenziano, in un immaginario "circolo virtuoso", gli studi clinici validati dalla Società Scientifiche Internazionali e/o le indicazioni assunte dalle Autorità Regolatorie, che attestano la capacità per i differenti sartani di esercitare, attraverso la modulazione del RAAS e il blocco selettivo recettoriale, sia l'azione antiipertensiva che la protezione dal danno d'organo e che, solo in alcuni casi e per alcuni di essi, hanno ottenuto la rimborsabilità dalle Autorità Regolatorie.

Lo schema grafico sintetizza graficamente l'intero spettro del rischio cardiovascolare nel paziente iperteso suddiviso in due settori principali: quello superiore relativo al rischio di eventi cardiaci (scompenso cardiaco e cardiopatia ischemica post-IMA) e quello inferiore agli eventi non cardiaci (ictus cerebrale e nefropatia). All'interno dello schema vengono collocati i grandi trial favorevoli che hanno documentato l'azione protettiva nei confronti dell'evento clinico. Per ogni azione documentata, viene inoltre indicato se l'indicazione ha ricevuto o meno la rimborsabilità da parte dell'Autorità Regolatoria.

\section{CONCLUSIONI}

Da quanto esposto si evince che la classe dei sartani si caratterizza per un'ampia eterogeneità sia per le proprietà farmacologiche sia per la documentazione clinica a sostegno delle indicazioni delle singole molecole. Quando per il trattamento di una data patologia sia disponibile ai medici un'ampia scelta di molecole all'interno della stessa classe farmacologica, e questo caso ne è un esempio, è auspicabile a nostro parere che i professionisti, rispettando le peculiarità dei singoli casi, rimangano il più possibile costanti nella scelta prescrittiva, così da assumere un'esperienza col farmaco e i suoi effetti terapeutici e collaterali. In aggiunta a tale aspetto i medici dovrebbero essere messi nella condizione di poter considerare quali siano le molecole che consentono di trattare il maggior 
numero di danni d'organo o comorbilità alle quali il paziente, in questo caso quello iperteso, può essere sottoposto nell'ambito della sua patologia di base.

Per quanto qui presentato risulta chiaro che nel caso dell'ipertensione e della terapia con sartani tale esigenza non può essere soddisfatta dalla disponibilità di un'unica molecola, ma ne richiede la presenza nel prontuario terapeutico a disposizione dei medici di almeno due; a questo proposito sommando la combinazione delle indicazioni terapeutiche desunte dalle linee guida Nazionali ed Internazionali con le "indicazioni approvate con rimborsabilità" dall'Autorità Regolatoria, almeno la disponibilità contemporanea di losartan e valsartan appare attualmente quella che più compiutamente soddisfa entrambe tali caratteristiche (Figura 10).

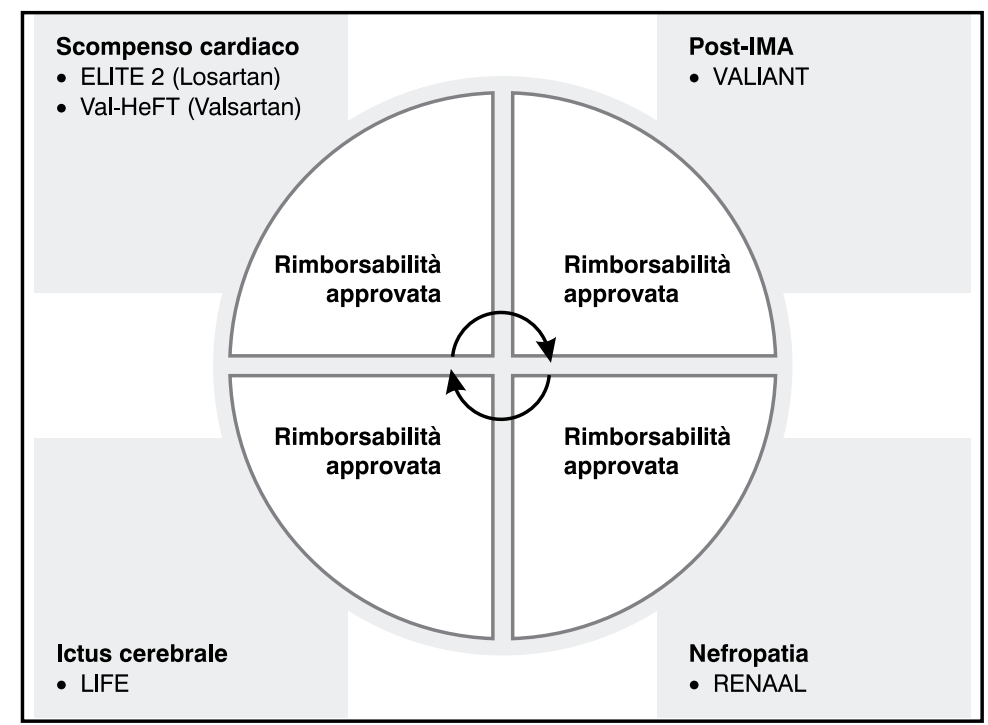

Figura 10

Circolo virtuoso di valsartan +losartan

\section{BIBLIOGRAFIA}

1. Unger T, Stoppelhaar M. Rationale for double renin-angiotensin-aldosterone system blockade. Am J Cardiol 2007; 100: $25 \mathrm{~J}-31 \mathrm{~J}$

2. Siragy HM. Angiotensin receptor blockers: how important is selectivity? Am J Hypertens 2002; 15: 1006-14

3. Mancia G, De Backer G, Dominiczak A, Cifkova R, Fagard R et al. 2007 Guidelines for the Management of Arterial Hypertension: The Task Force for the Management of Arterial Hypertension of the European Society of Hypertension (ESH) and of the European Society of Cardiology (ESC). J Hypertension 2007; 25: 1105-87 